\title{
Analysis of the breast cancer methylome using formalin-fixed paraffin-embedded tumour.
}

Ee Ming Wong ${ }^{1 \#}$, JiHoon E Joo ${ }^{1 \#}$, Catriona A McLean², Laura Baglietto ${ }^{3,4,5,6}$, Dallas R English $^{5,6}$, Gianluca Severi ${ }^{3,4,5,6,7}$, Hui-Chen $\mathrm{Wu}^{8}$, Mary Beth Terry ${ }^{9}$, John L Hopper ${ }^{6}$, Roger L Milne $^{5,6}$, Graham G Giles ${ }^{5,6}$, Melissa C Southey ${ }^{1,5 *}$.

1. Genetic Epidemiology Laboratory, Department of Pathology, The University of Melbourne, Victoria 3010, Australia

2. Anatomical Pathology, Alfred Health, The Alfred Hospital, Victoria 3181, Australia

3. Université Paris-Saclay, Univ. Paris-Sud, UVSQ, CESP, INSERM, Villejuif, France

4. Gustave Roussy, F-94805, Villejuif, France

5. Cancer Epidemiology Centre, Cancer Council Victoria, Melbourne, Australia

6. Centre for Epidemiology and Biostatistics, University of Melbourne, Melbourne, Victoria, Australia

7. HuGeF, Human Genetics Foundation, 10126 Torino, Italy

8. Department of Environmental Health Sciences, Mailman School of Public Health, Columbia University, New York, NY, USA

9. Department of Epidemiology, Mailman School of Public Health, Columbia University, New York, NY, USA

${ }^{\#}$ Equal contribution

*Author for correspondence:

Professor Melissa C. Southey 
Email: msouthey@unimelb.edu.au

Telephone: +6183444895

Fax: +6183444004

\begin{abstract}
Aberrant DNA methylation occurs frequently in breast carcinogenesis. Tools for translational epigenetic studies of breast cancer involving formalin-fixed paraffin-embedded (FFPE) human tissues have now been developed. Few studies have measured genome-wide methylation in DNA derived from paraffin-embedded tumour tissues and compared the DNA methylation in corresponding adjacent non-tumour ductal epithelium $\left(\mathrm{ADJ}_{\mathrm{NT}}\right)$. These studies are technically challenging due to; the spectrum of breast cancer pathologies, the variable suitability of DNA extracted from FFPE material and the difficulties in identifying $\mathrm{ADJ}_{\mathrm{NT}}$. We assessed the suitability of FFPE breast cancer material for genome-wide DNA methylation assessment of tumour and $\mathrm{ADJ}_{\mathrm{NT}}$. Twenty-one archival breast tumour tissues with paired $\mathrm{ADJ}_{\mathrm{NT}}$ obtained from separate blocks and at least $2 \mathrm{~cm}$ from the tumour were sourced from The Melbourne Collaborative Cohort Study (MCCS). DNA was prepared from macrodissected tissue samples and assessed for genome-wide methylation using the Infinium HumanMethylation450 Beadchip (HM450K) array. The 1,000 most differentially methylated probes between tumour and $\mathrm{ADJ}_{\mathrm{NT}}$ tissues in this FFPE-derived dataset differentiated tumour and $\mathrm{ADJ}_{\mathrm{NT}}$ tissues in The Cancer Genome Atlas Network data (TCGA; derived from high molecular weight DNA using the same HM450K array). Large-scale studies of genome-wide DNA methylation using FFPE breast cancer specimens offer the opportunity to further refine the pathological classification of tumours, to include subtypes that are underrepresented in the TCGA data and provide the capacity to further explore intra-tumoural heterogeneity.
\end{abstract}


Keywords Breast cancer, FFPE, methylome.

\section{Abbreviations}

$\mathrm{ADJ}_{\mathrm{NT}} \quad$ adjacent non-tumour ductal epithelium

$\beta \quad$ Beta

$\mu \mathrm{g} \quad$ microgram

$\mu 1 \quad$ microliter

$\mu \mathrm{m} \quad$ micron

$\mathrm{Cq} \quad$ quantitation cycle

DMP differentially methylated probe

FCD fibrocystic disease

FFPE formalin-fixed paraffin-embedded

H\&E haematoxylin and eosin

HIN-1 high in normal-1

HM450K Infinium HumanMethylation450 Beadchip

IDC infiltrating ductal carcinoma

MCCS Melbourne Collaborative Cohort Study

ng nanogram

NKX6-2 NK6 Homeobox 2

NOS not otherwise specified

QC quality control

qPCR quantitative polymerase chain reaction

RASSF1A RAS association domain family protein $1 A$

SWAN Subset-quantile Within Array Normalisation

TCGA The Genome Cancer Genomic Atlas Network 


\section{Introduction}

DNA methylation plays a critical role in cancer. Virtually all tumour cells feature genomic instability induced through global loss of DNA methylation and transcriptional silencing of tumour suppressor genes from promoter hypermethylation [1]. DNA methylation aberrations at several tumour suppressor genes have been measured in breast tumours and some of these methylation marks may explain specific clinicopathological characteristics [2]. Importantly, DNA methylation profiles may enable stratification over and beyond gene expression data. DNA methylation is more stable than gene transcripts and therefore more feasible for clinical use. For example, Dedeurwaerder et al (2011) have identified novel DNA methylation profiles that suggest existence of additional breast cancer subtypes above those classified by current expression subtypes [3].

Recent data from TCGA has dramatically expanded the number of genes identified as aberrantly methylated in breast cancers and several studies have found that DNA methylation might be responsible for at least some of the observed tumour heterogeneity $[4,5]$. Further, DNA methylation marks are dynamic and change throughout tumour progression [2]. Hoesel et al (2013) reported DNA hypermethylation of selected genes such as RASSF1A and RAR $\beta 2$ occur early in breast carcinogenesis suggesting the potential of their use as biomarkers for early detection [6]. Taken together, the potential for DNA methylation marks to be utilised as diagnostic and therapeutic targets in breast cancer is well established.

Recent advances in technology have enabled research in this area to expand from single or few molecularly-targeted assays to platforms that conduct genome-wide assessment of the methylome. The capacity of these platforms to scan the genome in one assay and their demonstrated utility for the analysis of FFPE material offer great promise to further refine our 
understanding of DNA methylation in cancer, including its role in neoplastic change and tumorigenic pathways of cancer progression [7]. Fulfilling this promise could enable the resources of large epidemiological studies, that commonly collect and store diagnostic FFPE material, to underpin ongoing research and identify new diagnostic and therapeutic agents and provide the evidence base for translation.

For some research questions there is great importance and reliance on assessing methylation in both tumour and adjacent non-tumour ductal epithelium $\left(\mathrm{ADJ}_{\mathrm{NT}}\right)$ for alterations in the tumorigenic process. The utility of this approach has been demonstrated by several studies that have primarily used loci-specific assessment of DNA extracted from fresh frozen material [8-11]. For example, Bardowell et al (2013) assessed the methylation levels of 70 candidate genes (across $1200 \mathrm{CpG}$ dinucleotides) in fresh frozen tumour and matched $\mathrm{ADJ}_{\mathrm{NT}}$ breast tissues and identified six distinct methylation patterns in these breast tumours, with some displaying correlated methylation-gene expression patterns. Li and colleagues measured DNA methylation in 48 genes from 150 paired fresh frozen breast tumour and $\mathrm{ADJ}_{\mathrm{NT}}$ tissue samples and reported a methylation pattern which could be used to predict the onset of breast cancer with specific clinicopathological features. Using pyrosequencing technology, Rauscher et al. (2015) showed that large field effects were present in breast cancer when they pyrosequenced 16 gene loci in more than 100 FFPE breast tumours, ADJ $_{\mathrm{NT}}$ breast tissue and unaffected control reduction mammoplasty samples. This observation was confirmed by the most comprehensive study of this type to date by Teschendorff et al (2016) who measured genome-wide methylation using the Infinium HumanMethylation450 Beadchip (HM450K) array from DNA extracted from snap-frozen breast cancer tissues, normal/benign tissues and matched breast and $\mathrm{ADJ}_{\mathrm{NT}}$ tumour tissues (total $\mathrm{n}=569$ ). 
Selecting tissue for analysis is challenging as it is complicated by several aspects related to the histological variation of breast cancers that result in: i) tumour-enriched DNA samples being prepared for analysis (variably contaminated with non-tumour material); ii) contamination of $\mathrm{ADJ}_{\mathrm{NT}}$ tissue with tumour cells; and iii) availability (identification) of histopathologically normal tissue from the adjacent fields. Work involving the use of FFPE material offers increased capacity for precision in regard to tissue selection, but the DNA extracted from this material is a more complex template (in terms of quality) for the current, high density molecular platforms.

In contrast to the work described above, we sought to evaluate the capacity of diagnostic FFPE material to provide DNA samples representing the tumour and $\mathrm{ADJ}_{\mathrm{NT}}$ breast tissue suitable for assessment on the HM450K array. We utilised resources from the MCCS and measured genome-wide methylation in DNA extracted from 21 pairs of breast tumour and $\mathrm{ADJ}_{\mathrm{NT}}$ tissue.

\section{Materials and Methods}

\section{Melbourne Collaborative Cohort Study (MCCS)}

The MCCS is a prospective study of more than 40,000 men and women who were recruited in the years 1990-1994 [12]. The vast majority of participants (>99\%) were aged 40-69 at recruitment (baseline), when a wide range of epidemiological data was collected from the participants as well as blood samples and physical measurements. Pathology materials related to breast cancers diagnosed in female participants were retrieved from the diagnostic service laboratory and reviewed by qualified pathologists. Representative haematoxylin and eosin (H\&E) stained sections and unstained sections $(3 \mu \mathrm{m})$ were prepared and stored desiccated at $4{ }^{\circ} \mathrm{C}$ for up to 15 years. Immunohistochemical staining and breast cancer subtyping were 
conducted as described by Blows et al (2010) [13]. Written informed consent was obtained from each participant and the study was approved by the Human Research Ethics Committee of Cancer Council Victoria.

\section{DNA extraction from FFPE breast tumour and ADJ $\mathbf{J}_{\mathrm{NT}}$ tissues}

DNA was extracted as previously described from FFPE breast tumour and $\mathrm{ADJ}_{\mathrm{NT}}$ material that had been fixed in separate tissue blocks [7]. Briefly, the tumour area and the $\mathrm{ADJ}_{\mathrm{NT}}$ tissue for 21 individuals (42 FFPE tissue samples in total) most suitable for macrodissection was identified by a single pathologist (CAM) using the WHO Classification of Tumours of the Breast criteria [14]. FFPE tissue was macrodissected from at least two $3 \mu \mathrm{m}$ methylGreen-stained sections using a 21-guage needle followed by protease digestion using Proteinase K for 48 hours. Macrodissection for all FFPE tissue samples was performed on the same day by the same individual (EMW) to reduce potential batch effects. DNA was extracted using the QIAamp DNA FFPE tissue kit (Qiagen, Hilden, Germany) as per manufacturer's protocol except that DNA was eluted twice to obtain a final volume of $25 \mu 1$ and stored at $4{ }^{\circ} \mathrm{C}$.

\section{Sodium bisulfite conversion}

Sodium bisulfite conversion was performed using the EZ-DNA Methylation-Gold kit (Zymo, Irvine, CA, USA). FFPE-derived DNA was bisulfite converted (24 - 750 ng input DNA) and eluted in a final volume of $8 \mu$ l elution buffer. Bisulfite converted DNA was stored long-term at $-20^{\circ} \mathrm{C}$.

\section{Quality control (QC) assessments}


The following QC assessments were applied to extracted FFPE DNA samples prior to the application on the HM450K array: 1) QC1: confirm the availability of DNA after extraction using the Qubit dsDNA BR assay (Life Technologies, Carlsbad, CA, USA); 2) QC2: evaluate the quality of archival tissue DNA using the Infinium HD FFPE QC assay (Illumina, San Diego, CA, USA); and 3) QC3: confirm the availability of DNA after sodium bisulfite conversion and FFPE restoration using an in-house bisulfite-specific qPCR assay [7].

\section{Infinium HumanMethylation450 Beadchip (HM450K) assay}

The HM450K assay (Illumina, San Diego, CA, USA) was performed as per manufacturer's instructions. One FFPE tumour and $\mathrm{ADJ}_{\mathrm{NT}}$ DNA pair were run as replicates to ensure the reproducibility of data and one high molecular weight DNA extracted from a U266 multiple myeloma cell line was included to further control the assay.

\section{Data processing and statistical analysis}

Raw data was imported into $\mathrm{R}$ environment, normalised and processed using the minfi package as previously described $[15,16]$. HM450K probes or individual samples that did not meet the following criteria were removed from further analysis: 1) average detection p-value across all probes/sample $\leq 0.01 ; 2$ ) individual probe detection $\mathrm{p}$-value of $\leq 0.05$. $\beta$ and $\mathrm{M}$ values were calculated from 480,136 CpG probes in 36 samples. Three-dimensional principal component analysis (3D-PCA) plots were generated using the scatterplot3d $\mathrm{R}$ package. Differentially methylated probes were identified using the limma Bioconductor package [17], taking the pairwise design into account. All graphics were generated using ggplot 2 unless stated otherwise. 


\section{Analysis of TCGA data}

The HM450K data of matched tumour and $\mathrm{ADJ}_{\mathrm{NT}}$ tissues (> $2 \mathrm{~cm}$ from tumour) from 90 women was downloaded from the National Cancer Institute webpage (https://tcgadata.nci.nih.gov/tcga/tcgaDownload.jsp). The data was background corrected and normalised according to the Illumina and Subset-quantile Within Array Normalization (SWAN) methods [18]. The clinical information was also downloaded from the NCI website.

\section{Comparison with other tumour-ADJ ${ }_{\text {NT }}$ studies}

To test for the age-associated methylation in normal breast tissue as reported by Johnson et al (2014) [19], linear regression was performed using M-values from the $18 \mathrm{ADJ}_{\mathrm{NT}}$ tissues and patient age, which ranged from 51.7 to 77.6 years [17]. Differentially Methylated Regions (DMRs) were identified between the 18 tumour and ADJ NT $_{\text {N }}$ pairs using the DMRcate $\mathrm{R}$ package $[20]$ and individually compared with the 37 differentially methylated gene promoters reported by Li et al (2015) [9].

\section{Results}

Histological examination of the FFPE breast material collected from 21 breast cancer cases participating in the MCCS identified tumour enriched and $\mathrm{ADJ}_{\mathrm{NT}}$ areas for macrodissection. The $\mathrm{ADJ}_{\mathrm{NT}}$ areas included nine cases with histologically normal duct epithelium $(\mathrm{N})$, six cases with atrophic duct tissue and three cases with fibrocystic disease (FCD) (Table 1). The estimated percentage of normal ductal epithelium on a background of stroma and adipose components ranged from $3-10 \%$. The tumours comprised $>99.5 \%$ infiltrating tumour in all cases. Ductal Carcinoma In Situ constituted $<0.5 \%$ of macrodissected material. 
The amount of DNA extracted from these 42 tumour and $\mathrm{ADJ}_{\mathrm{NT}}$ FFPE tissue samples (21 pairs) ranged from $24 \mathrm{ng}$ to $1.5 \mathrm{ug}$. Due to insufficient DNA, one $\mathrm{ADJ}_{\mathrm{NT}}$ sample and its matched tumour DNA were not progressed further. The remaining 40 tumour and $\mathrm{ADJ}_{\mathrm{NT}}$ DNA samples passed the Infinium HD QC assay threshold cutoff of $\Delta \mathrm{Cq} \leq 6$. After sodium bisulfite conversion and FFPE restoration, two $\mathrm{ADJ}_{\mathrm{NT}}$ tissue DNA samples did not meet the final bisulfite-specific qPCR threshold cutoff $(\Delta \mathrm{Cq} \geq 4)$ and together with their matched tumour DNA were not assayed on the HM450K array [7]. A total of 36 FFPE tumour and $\mathrm{ADJ}_{\mathrm{NT}}$ DNA samples from 18 individuals remained for genome-wide methylation profiling on the HM450K array.

\section{Genome-wide methylation profiles}

After removing probes that did not pass the HM450K data quality criteria, 480, 136 Infinium probes $(98.9 \%)$ remained for further analysis. Three-dimensional PCA was performed to illustrate overall similarities based on DNA methylation levels detected by all remaining CpG probes. Figure 1 shows the relationship between all tumour and $\mathrm{ADJ}_{\mathrm{NT}}$ samples and also the relationship between each tumour and its matching $\mathrm{ADJ}_{\mathrm{NT}}$ sample from each of the 18 women. Supplementary Figure 1 shows the scatterplots between all 18 tumour and ADJ pairs and their respective correlation coefficients. Figure 2 further illustrates sample $3(\mathrm{R}=$ 0.984) and sample $7(\mathrm{R}=0.853)$.

\section{Comparison of FFPE genome-wide methylation with TCGA}

We compared the FFPE HM450K dataset with the publicly available TCGA HM450K dataset. A total of 105,545 marks were differentially methylated $\left(\right.$ FDR $_{\text {adj }} \mathrm{p}$-value $\left.\leq 0.01\right)$ between the archival FFPE tumour and $\mathrm{ADJ}_{\mathrm{NT}}$ DNAs. Of these, 101,672 (> $96 \%$ ) marks were also differentially methylated in the TCGA dataset (data not shown). To test whether 
the methylation status of DMPs identified from this FFPE dataset could also separate tumour samples from matched $\mathrm{ADJ}_{\mathrm{NT}}$ tissues in TCGA dataset (generated from fresh frozen material), we plotted a methylation heatmap using the 1000 most significantly DMPs identified in the FFPE dataset. We found that the DMPs were effective for differentiating tumour and $\mathrm{ADJ}_{\mathrm{NT}}$ tissues within the TCGA dataset (Figure 3).

We further compared the DNA methylation levels between the FFPE-derived samples and fresh frozen samples from TCGA in selected genes. The RAS association domain family protein $1 A(R A S S F 1 A)$ and high in normal-1 (HIN-1) genes were assessed as these tumour suppressor genes have been shown to often be deregulated through methylation aberration in breast cancer. The NK6 Homeobox 2 (NKX6-2) gene was assessed as it was identified to be one of most significant differentially methylated regions (clusters of $\mathrm{CpG}$ sites) in the FFPEderived samples. For all three genes, methylation profiles observed in both the FFPE and TCGA datasets were highly correlated (Supplementary Figure 2).

\section{Comparison of FFPE genome-wide methylation with other tumour-ADJNT studies}

Johnson et al (2014) reported $24 \mathrm{HM} 27 \mathrm{~K}$ probes with age-related methylation in normal breast tissue were also significantly differentially methylated between normal and breast tumour tissues [19]. While cg15465321 probe was not available in the HM450K array, the $\underline{\text { FFPE data demonstrated statistically significant }\left(\mathrm{FDR}_{\text {adi. }} \text { p-value }<0.05\right) \text { differential }}$ methylation at 17 of the remaining 23 probes reported by Johnson et al (Supplementary Figure 3). Although we did not identify any significant probes associated with age in the $\mathrm{ADJ}_{\mathrm{NT}}$ tissues, this was likely to be due to the lack of power. 
Using microfluidic PCR-based target enrichment and next-generation bisulfite sequencing technology, Li et al (2015) analyzed methylation status of 48 candidate genes in paired tumour and normal tissues from 180 Chinese breast cancer patients [9]. The promoter regions of 31 of the 37 genes reported by Li et al. were also differentially methylated in the FFPEderived dataset (Supplementary table 1). Interestingly, SOX17 that had been identified as one of the most significant candidate biomarker for cancer prediction had one of the smallest $\underline{\text { Stouffer-transformed FDR adjusted p-value in the FFPE dataset }(F D R}$ adi. $\underline{\text { p-value }}=4.17 \mathrm{X}$ $\left.10^{-39}\right)$.

\section{Discussion}

There is an increasing need to conduct large-scale studies examining genome-wide DNA methylation of FFPE breast cancer specimens as it offers opportunities to increase histological precision, to study subtypes that are underrepresented in the TCGA data and to provide capacity to further explore intra-tumoural heterogeneity. This report demonstrates the potential of FFPE material to be used in this setting. Although TCGA has comprehensive and systematic analyses of molecular changes such as DNA methylation for 707 breast tumours [4], there are several limitations for its clinical use: 1) there is missing molecular subtype information on $37 \%$ of TCGA breast cancer cases; 2) family history was not collected in TCGA, and there is incomplete clinical information on many of the cases making it impossible to stratify the results for clinical counseling based on underlying risk of breast cancer; and 3) only $13 \%$ of samples have methylation profiles in the corresponding nontumour tissues. As the epigenomes vary according to characteristics of the study sample including the distribution of age, race/ethnicity and underlying risk, it is essential to select a well characterized sample for the identification of markers so the results will be useful for 
integration into clinical care [21]. Thus it is critical to know how viable FFPE methods are when applying them to large epidemiologic cohorts.

The comparison of this FFPE DNA-derived HM450K data with TCGA HM450K data demonstrated consistencies between the two datasets despite the differences in sample size and DNA source. By applying the 1000 most DMPs in the FFPE dataset to TCGA data from fresh frozen material, we found that the same DMPs were able to differentiate $\mathrm{ADJ}_{\mathrm{NT}}$ from tumour tissue in TCGA dataset (Figure 3). Further demonstration of the reliability of the FFPE methylation data was achieved by comparison of the data with the studies of Johnson et al (2014) and Li et al (2015) $[9,19]$.

We additionally performed a simple methylation assessment of several genes widely reported to be aberrantly methylated in cancer. Both RASSFIA and HIN-1 are tumour suppressor genes that are frequently methylated in breast cancer [6,22-24]. In RASSF1A, only one of the two CpG islands in the promoter region is methylated in breast cancer tissue [24,25]. Evaluation of the Infinium probes across the RASSF1A promoter confirmed that the probes overlapping this $\mathrm{CpG}$ island (chromosome 3: 50,377,815 - 50,378,540) had high levels of methylation in the FFPE-derived breast tumours (Supplementary Figure 2). Evaluation of the FFPE data found the NKX6-2 gene to be differentially methylated in tumour compared with $\mathrm{ADJ}_{\mathrm{NT}}$ tissues across all probes specific to the gene (Individual probe $\Delta \beta=0.02-0.45$ ). NKX6-2 is a homeobox gene that has previously been demonstrated to be methylated in renal cell and bladder cancer [26,21]. Methylation of this gene in breast cancer has not been reported previously. 
This work provides evidence that the resources of large collections of FFPE material could be used in studies examining the role of methylation in breast cancer tumourigenesis. This could allow epidemiological studies to underpin ongoing research aiming to identify new diagnostic markers and therapeutic agents and at the same time provide the evidence base for translation of such information.

\section{Acknowledgements and Funding Information}

This study was made possible by the contribution of many people including those taking part in the Melbourne Collaborative Cohort Study, the original investigators and the diligent team who manage the study and continue working on follow up. Cohort recruitment was funded by VicHealth and Cancer Council Victoria. This work was further supported by the National Health and Medical Research Council (NHMRC; APP1011618 and APP1026892) and The Victorian Breast Cancer Research Consortium. MCS is a NHMRC Senior Research Fellow JLH is a NHMRC Senior Principal Research Fellow. EMW is a recipient of the Dyason Fellowship.

\section{Author's Contributions}

EMW performed all the laboratory experiments described in this manuscript. EMW and JEJ performed the analysis of the experimental data. CAM contributed histopathological expertise. LB, DRE, MCS, GS and JLH made prior contribution to the MCCS resource utilised in this report. LB, MCS and DRE provided grant support for this study. RM contributed to the MCCS resource and logistical aspects of the project. GGG is the principal investigator of the Melbourne Collaborative Cohort Study. MCS provided additional grant support, the initial idea of the research described and overall supervision of the laboratory and analytical approaches. EMW, JEJ and MCS wrote the first draft of the manuscript to 
which all authors contributed. MBT and HCW contributed to manuscript preparation. The final manuscript was read and approved by all the authors.

\section{Conflict of interest}

The authors declare that they have no conflict of interest.

Ethical approval: “All procedures performed in studies involving human participants were in accordance with the ethical standards of the institutional and/or national research committee and with the 1964 Helsinki declaration and its later amendments or comparable ethical standards.

Informed consent: Informed consent was obtained from all participants in this research study.

\section{References}

1. Jones PA, Baylin SB (2002) The fundamental role of epigenetic events in cancer. Nat Rev Genet $3(6): 415-428$

2. Locke WJ, Clark SJ (2012) Epigenome remodelling in breast cancer: insights from an early in vitro model of carcinogenesis. Breast Cancer Res 14 (6):215. doi:10.1186/bcr3237

3. Dedeurwaerder S, Desmedt C, Calonne E (2011) Largest ever DNA methylation dataset for breast cancer completed. Expert Rev Mol Diagn 11 (5):470

4. Comprehensive molecular portraits of human breast tumours (2012). Nature 490 (7418):61-70. doi:10.1038/nature11412 
5. Stirzaker C, Zotenko E, Song JZ et al. (2015) Methylome sequencing in triple-negative breast cancer reveals distinct methylation clusters with prognostic value. Nat Commun 6:5899. doi:10.1038/ncomms6899

6. Jovanovic J, Ronneberg JA, Tost J et al. (2010) The epigenetics of breast cancer. Mol Oncol 4 (3):242-254. doi:10.1016/j.molonc.2010.04.002

7. Wong EM, Joo JE, McLean CA et al. (2015) Tools for translational epigenetic studies involving formalin-fixed paraffin-embedded human tissue: applying the Infinium HumanMethyation450 Beadchip assay to large population-based studies. BMC Res Notes 8:543. doi:10.1186/s13104-015-1487-z

8. Bardowell SA, Parker J, Fan C et al. (2013) Differential methylation relative to breast cancer subtype and matched normal tissue reveals distinct patterns. Breast Cancer Res Treat 142 (2):365-380. doi:10.1007/s10549-013-2738-0

9. Li Z, Guo X, Wu Y et al. (2015) Methylation profiling of 48 candidate genes in tumor and matched normal tissues from breast cancer patients. Breast Cancer Res Treat 149 (3):767779. doi:10.1007/s10549-015-3276-8

10. Rauscher GH, Kresovich JK, Poulin M et al. (2015) Exploring DNA methylation changes in promoter, intragenic, and intergenic regions as early and late events in breast cancer formation. BMC Cancer 15:816. doi:10.1186/s12885-015-1777-9 
11. Teschendorff AE, Gao Y, Jones A et al. (2016) DNA methylation outliers in normal breast tissue identify field defects that are enriched in cancer. Nat Commun 7:10478. doi:10.1038/ncomms 10478

12. Giles GG, English DR (2002) The Melbourne Collaborative Cohort Study. IARC Sci Publ 156:69-70

13. Blows FM, Driver KE, Schmidt MK et al. (2010) Subtyping of breast cancer by immunohistochemistry to investigate a relationship between subtype and short and long term survival: a collaborative analysis of data for 10,159 cases from 12 studies. PLoS Med 7 (5):e1000279. doi:10.1371/journal.pmed.1000279

14. WHO Classification of Tumours of the Breast (2012). 4th edn. International Agency for Research on Cancer (IARC), Lyon

15. Joo JE, Wong EM, Baglietto L et al. (2013) The use of DNA from archival dried blood spots with the Infinium HumanMethylation450 array. BMC Biotechnol 13:23. doi:10.1186/1472-6750-13-23

16. Aryee MJ, Jaffe AE, Corrada-Bravo H et al. (2014) Minfi: a flexible and comprehensive Bioconductor package for the analysis of Infinium DNA methylation microarrays. Bioinformatics 30 (10):1363-1369. doi:10.1093/bioinformatics/btu049

17. Ritchie ME, Phipson B, Wu D et al. (2015) limma powers differential expression analyses for RNA-sequencing and microarray studies. Nucleic Acids Res 43 (7):e47. doi:10.1093/nar/gkv007 
18. Maksimovic J, Gordon L, Oshlack A (2012) SWAN: Subset-quantile within array normalization for illumina infinium HumanMethylation450 BeadChips. Genome Biol 13 (6):R44. doi:10.1186/gb-2012-13-6-r44

19. Johnson KC, Koestler DC, Cheng C et al. (2014) Age-related DNA methylation in normal breast tissue and its relationship with invasive breast tumor methylation. Epigenetics 9 (2):268-275. doi:10.4161/epi.27015

20. Peters TJ, Buckley MJ, Statham AL et al. (2015) De novo identification of differentially methylated regions in the human genome. Epigenetics Chromatin 8:6. doi:10.1186/17568935-8-6

21. Chung W, Bondaruk J, Jelinek J et al. (2011) Detection of bladder cancer using novel DNA methylation biomarkers in urine sediments. Cancer Epidemiol Biomarkers Prev 20 (7):1483-1491. doi:10.1158/1055-9965.EPI-11-0067

22. Agathanggelou A, Honorio S, Macartney DP et al. (2001) Methylation associated inactivation of RASSF1A from region 3p21.3 in lung, breast and ovarian tumours. Oncogene 20 (12):1509-1518. doi:10.1038/sj.onc. 1204175

23. Krop IE, Sgroi D, Porter DA et al. (2001) HIN-1, a putative cytokine highly expressed in normal but not cancerous mammary epithelial cells. Proc Natl Acad Sci U S A 98 (17):97969801. doi:10.1073/pnas.171138398 
24. Yeo W, Wong WL, Wong N et al. (2005) High frequency of promoter hypermethylation of RASSF1A in tumorous and non-tumourous tissue of breast cancer. Pathology 37 (2):125130

25. Volodko N, Salla M, Zare A et al. (2016) RASSF1A Site-Specific Methylation Hotspots in Cancer and Correlation with RASSF1C and MOAP-1. Cancers (Basel) 8 (6). doi:10.3390/cancers8060055

26. Slater AA, Alokail M, Gentle D et al. (2013) DNA methylation profiling distinguishes histological subtypes of renal cell carcinoma. Epigenetics 8 (3):252-267. doi:10.4161/epi.23817

Fig 1 Genome-wide DNA methylation profiles tumour DNA (black) and $\mathrm{ADJ}_{\mathrm{NT}}$ (green) samples presented as a 3D-PCA plot. Tumour and $\mathrm{ADJ}_{\mathrm{NT}}$ samples are numbered as per Table 1. Dotted lines connect each matched tumour and $\mathrm{ADJ}_{\mathrm{NT}}$ pair.

Fig 2 Comparison between matched tumour and $\mathrm{ADJ}_{\mathrm{NT}}$ tissues for samples 3 and 7. a) Scatterplot of 480, 136 methylation probes between tumour (x-axis) and $\mathrm{ADJ}_{\mathrm{NT}}$ (y-axis) DNA. b) Tumour tissue (H\&E, X10) c) $\mathrm{ADJ}_{\mathrm{NT}}$ tissue (H\&E, X5).

Fig 3 Unsupervised clustering analysis of the 1000 most differentially methylated probes between tumour (T) and $\operatorname{ADJ}_{\mathrm{NT}}(\mathrm{N})$ DNA in the a) FFPE HM450K dataset and b) TCGA HM450K dataset. 
Sup Fig 1 Scatterplots and correlation coefficients for ADJ ${ }_{\text {NT }}$ (x-axis) and tumour (y-axis) pairs based on 480, 136 methylation probes.

Sup Fig 2 Comparison of Illumina probe methylation levels between the FFPE and TCGA datasets for a) $H I N-1$ and b) NKX6-2 and c) RASSF $1 A$.

Sup Fig 3 Scatterplots of 23 HM450K probes reported by Johnson et al (2014) to be differentially methylated between tumour and normal samples. * denotes probes that are significantly differentially methylated in the FFPE dataset between tumour (black) and $\underline{\mathrm{ADJ}}_{\mathrm{NT}}$ (red) samples (FDR $\underline{a d j}_{\text {. }}$-value $\left.<0.05\right)$.

Sup Table 1 Thirty-one of the 37 promoter regions reported to be differentially methylated between tumour and ADJ ${ }_{\text {NT }}$ samples in the FFPE dataset.

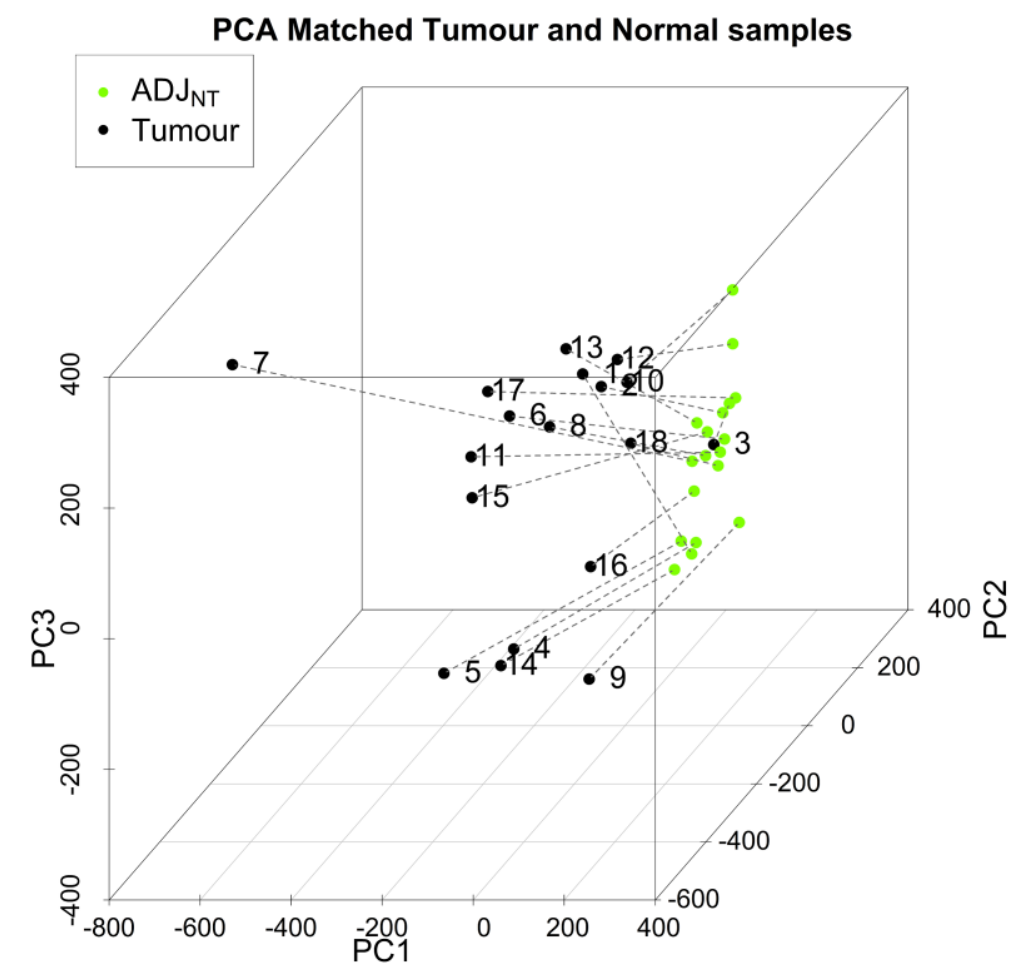

Fig 1 

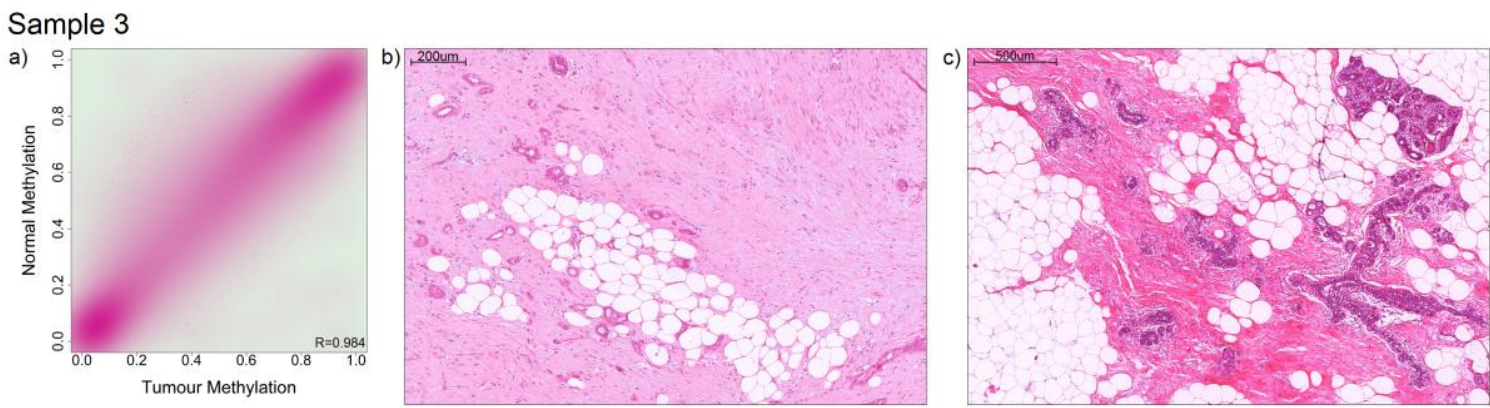

Sample 7
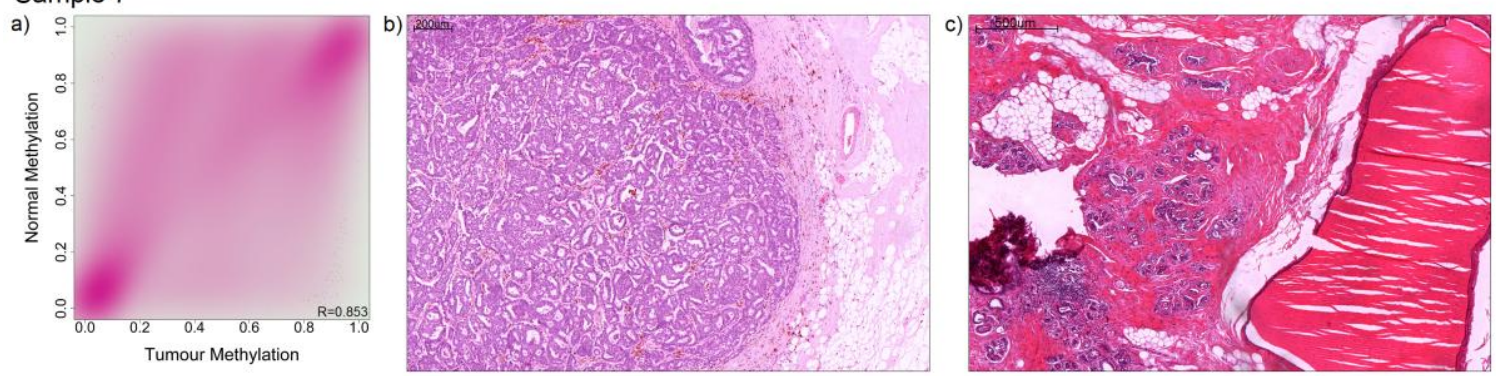

Fig 2
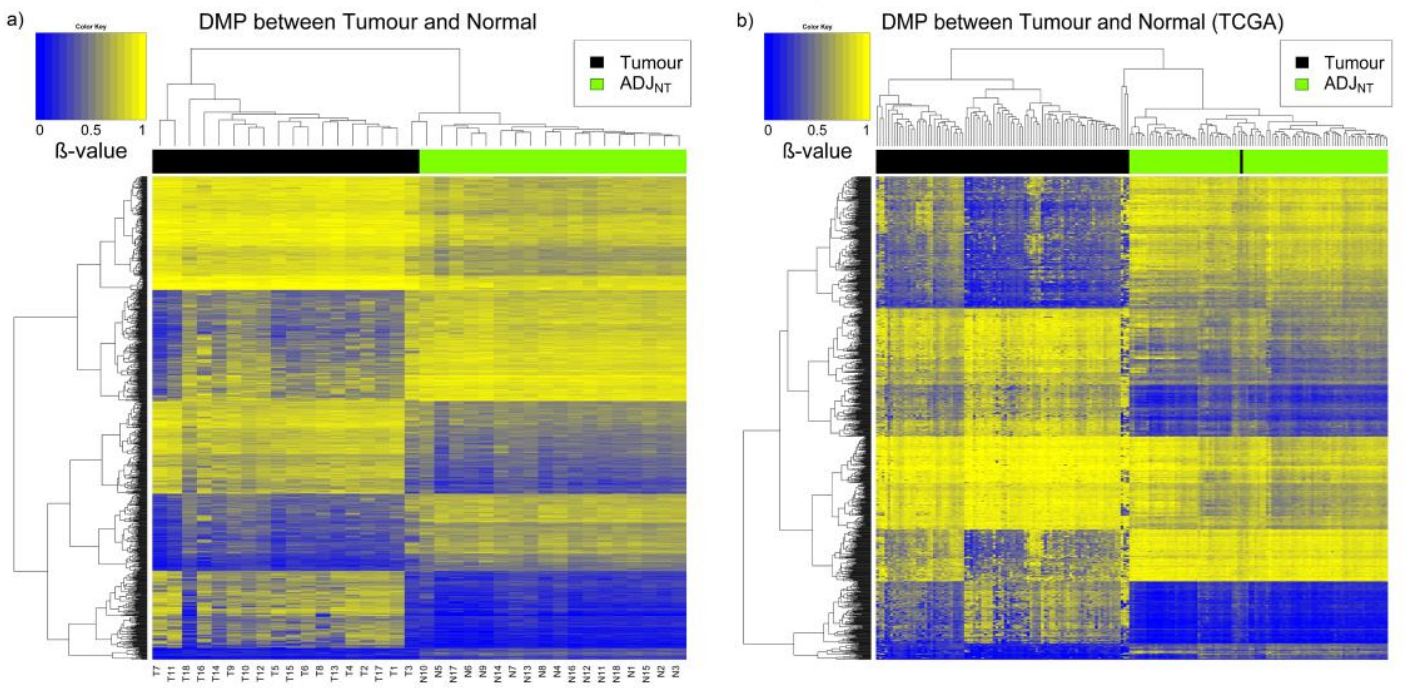

Fig 3

Table 1: Histological and molecular classification of tumour and $A D J_{\mathrm{NT}}$ tissue.

\begin{tabular}{|l|l|l|l|l|l|}
\hline Sample ID & $\begin{array}{l}\text { ADJ }_{\mathrm{NT}} \text { tissue } \\
\text { classification }\end{array}$ & $\begin{array}{l}\text { Tumour tissue } \\
\text { histological } \\
\text { classification }\end{array}$ & $\begin{array}{l}\text { Tumour tissue } \\
\text { molecular } \\
\text { subtype }\end{array}$ & $\begin{array}{l}\text { Tumour } \\
\text { grade }\end{array}$ & $\begin{array}{l}\text { Estrogen } \\
\text { receptor } \\
\text { status }\end{array}$ \\
\hline 1 & $\mathrm{~N}$ & IDC, NOS & Luminal A & 2 & Negative \\
\hline 2 & Atrophic duct & IDC, NOS & Luminal A & 1 & Positive \\
\hline 3 & N & IDC, NOS & Luminal A & 1 & Positive \\
\hline
\end{tabular}




\begin{tabular}{|l|l|l|l|l|l|}
\hline 4 & N & IDC, NOS & Luminal A & 3 & Positive \\
\hline 5 & Atrophic duct & IDC, NOS & Luminal A & 3 & Positive \\
\hline 6 & N & IDC, NOS & Luminal A & 3 & Positive \\
\hline 7 & FCD & IDC, NOS & Luminal A & 1 & Positive \\
\hline 8 & Atrophic duct & IDC, NOS & Luminal A & 2 & Positive \\
\hline 9 & N & IDC, NOS & HER2-enriched & 3 & Negative \\
\hline 10 & FCD & IDC, NOS & Luminal A & 2 & Positive \\
\hline 11 & N & IDC, NOS & Luminal A & 1 & Positive \\
\hline 12 & N & IDC, NOS & Luminal A & 2 & Positive \\
\hline 13 & Atrophic duct & IDC, NOS & Luminal A & 2 & Positive \\
\hline 14 & N & Tubular & Luminal A & 1 & Positive \\
\hline 15 & N & IDC, NOS & Luminal A & 2 & Negative \\
\hline 16 & Atrophic duct & IDC, NOS & TN & 2 & Negative \\
\hline 17 & Atrophic duct & Lobular, classical & Luminal A & 2 & Positive \\
\hline 18 & FCD & Mucinous, colloid & Luminal A & 1 & Positive \\
\hline
\end{tabular}

FCD: Fibrocystic disease; IDC: Infiltrating ductal; NOS: Not Otherwise Specified;

TN: Triple negative (ER $\left./ \mathrm{PR}^{-} / \mathrm{HER}^{-}{ }^{-}\right)$; $\mathrm{N}$ : histologically normal breast epithelium. 
Sup Table 1 Thirty-one of the 37 promoter regions reported to be differentially methylated betwee region, ${ }^{3}$ Minimum FDR adj. $p$-value of the region, ${ }^{4}$ Stouffer transformation of FDR adj $p$-vals of in

\begin{tabular}{|c|c|c|c|}
\hline Chromosome & Start coordinate & Stop coordinate & \# CpGs ${ }^{1}$ Promoters ${ }^{2}$ \\
\hline chr2 & 211089678 & 211090487 & 10 ACADL-001, ACADL-003, \\
\hline chr9 & 18473902 & 18474243 & 2 ADAMTSL1-005, ADAMTSL \\
\hline chr5 & 112073686 & 112074043 & 3 APC-001, APC-004, APC-003, \\
\hline chr6 & 7728676 & 7729794 & 5 BMP6-001 \\
\hline chr11 & 115371789 & 115373119 & 6 CADM1-007, CADM1-008 \\
\hline chr11 & 115088647 & 115088907 & 4 CADM1-016 \\
\hline chr7 & 116164108 & 116165941 & 21 CAV1-001, CAV1-003, CAV \\
\hline chr12 & 4378267 & 4384890 & 61 CCND2-001, CCND2-004, C \\
\hline chr7 & 94023308 & 94024254 & 11 COL1A2-001 \\
\hline chr5 & 149492480 & 149493814 & 8 CSF1R-001, CSF1R-004, PL \\
\hline chr1 & 68515209 & 68517691 & 24 DIRAS3-001, DIRAS3-201, \\
\hline chr7 & 55086091 & 55088104 & 8 EGFR-001, EGFR-003, EGF \\
\hline chr7 & 55177182 & 55177623 & 2 EGFR-009, EGFR-202 \\
\hline chr6 & 152125861 & 152130332 & 44 ESR1-001, ESR1-002, ESR \\
\hline chr6 & 152011103 & 152011666 & 7 ESR1-004, ESR1-201 \\
\hline chr6 & 152200760 & 152201611 & 4 ESR1-010 \\
\hline chr11 & 67349309 & 67352041 & 19 GSTP1-004, GSTP1-001, G \\
\hline chr17 & 1957529 & 1958237 & 25 HIC1-005, HIC1-006, HIC1-0 \\
\hline chr11 & 534232 & 534632 & 4 HRAS-002, HRAS-004, HRA \\
\hline chr19 & 10397031 & 10399157 & 10 ICAM5-001, ICAM4-001, ICA \\
\hline chr11 & 536758 & 537737 & 13 LRRC56-001, HRAS-002, HF \\
\hline chr7 & 24323128 & 24325371 & 15 NPY-001, NPY-002, NPY-003 \\
\hline chr5 & 149535646 & 149535765 & 4 PDGFRB-001, PDGFRB-004, \\
\hline chr5 & 149512117 & 149513303 & 4 PDGFRB-009 \\
\hline chr17 & 56832804 & 56833425 & 13 PPM1E-001 \\
\hline chr11 & 6340283 & 6342510 & 17 PRKCDBP-001, PRKCDBP-( \\
\hline chr1 & 186649153 & 186650479 & 15 PTGS2-001, PTGS2-002, PT \\
\hline chr9 & 36035988 & 36037454 & 6 RECK-001, RECK-003, REC \\
\hline chr1 & 27188505 & 27190639 & 13 SFN-001 \\
\hline chr8 & 41164679 & 41168336 & 23 SFRP1-001, SFRP1-003 \\
\hline chr4 & 154709441 & 154714852 & 55 SFRP2-001 \\
\hline chr8 & 55370192 & 55372569 & 18 SOX17-001 \\
\hline chr5 & 1282357 & 1283350 & 7 TERT-003 \\
\hline chr5 & 1266145 & 1266595 & 3 TERT-007 \\
\hline chr5 & 1293871 & 1296007 & 10 TERT-201, TERT-001, TER 1 \\
\hline chr3 & 30646532 & 30648407 & 16 TGFBR2-001, TGFBR2-002 \\
\hline chr22 & 33195343 & 33197394 & 16 TIMP3-001 \\
\hline chr8 & 23020966 & 23022403 & 9 TNFRSF10D-001 \\
\hline chr12 & 65514473 & 65516002 & 9 WIF1-001, WIF1-002 \\
\hline chr8 & 30892276 & 30892490 & 4 WRN-001, PURG-001, PUR \\
\hline chr8 & 30888801 & 30890632 & 15 WRN-001, PURG-001, PUR \\
\hline
\end{tabular}



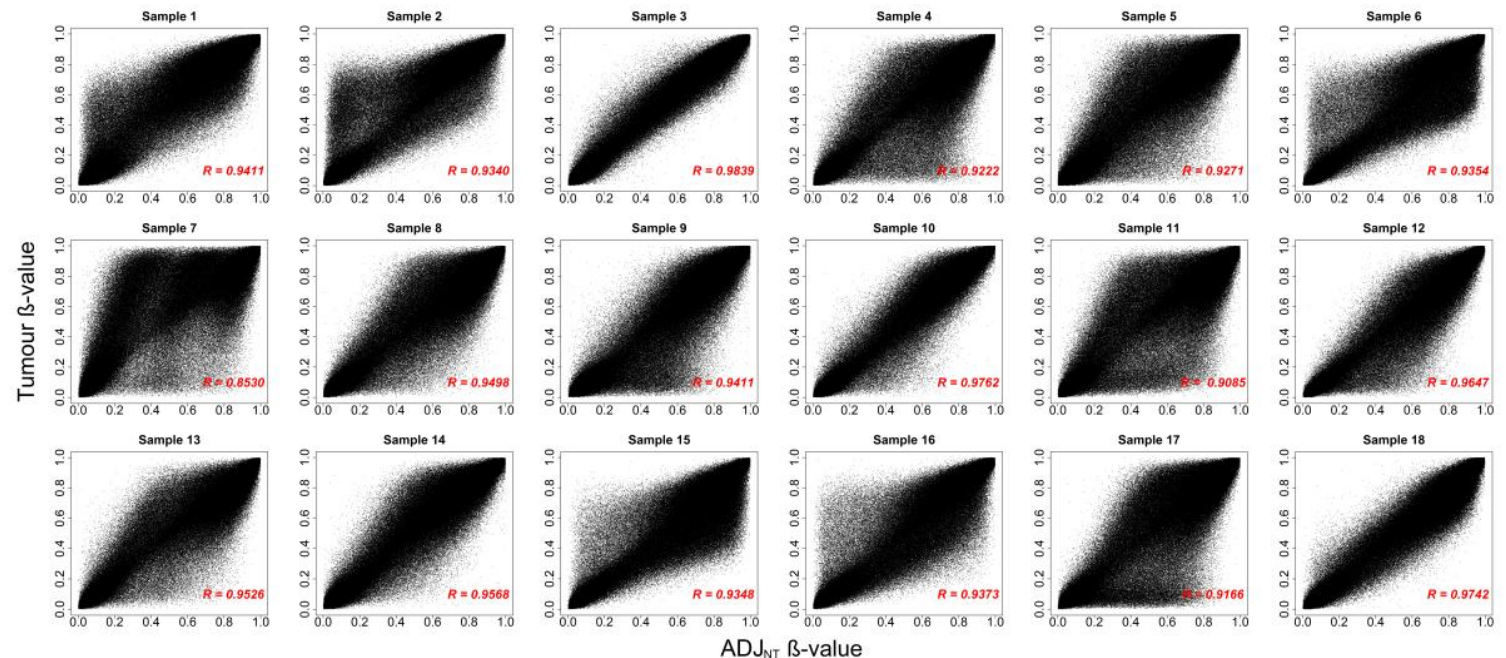

Sup Fig 1 
a) $\mathrm{HIN}-1$

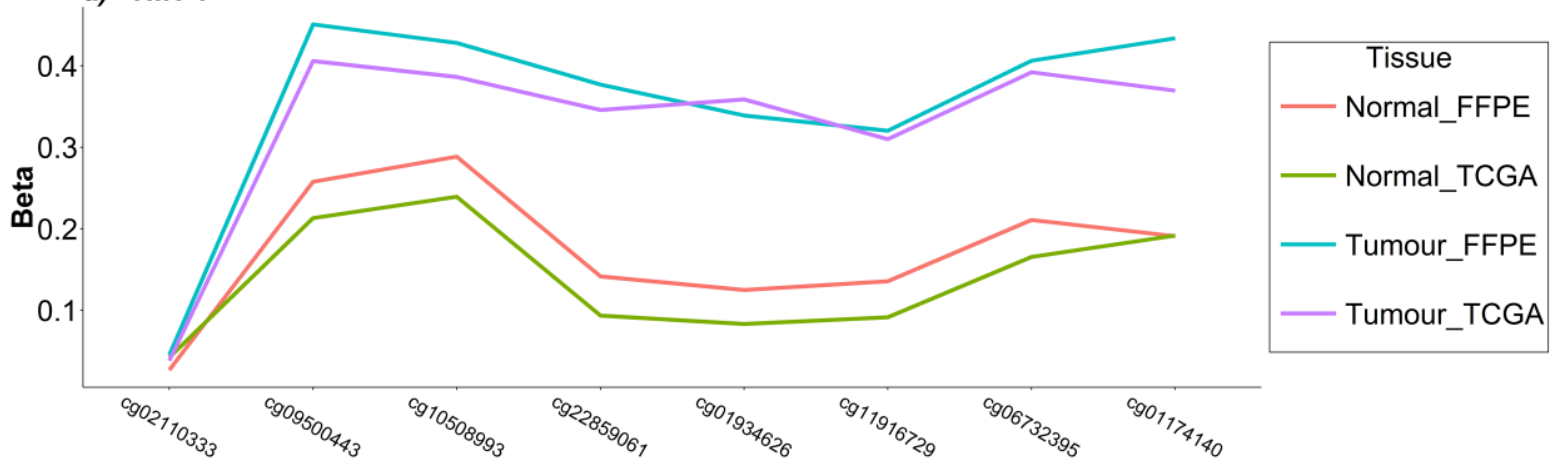

b) $N K X 6-2$
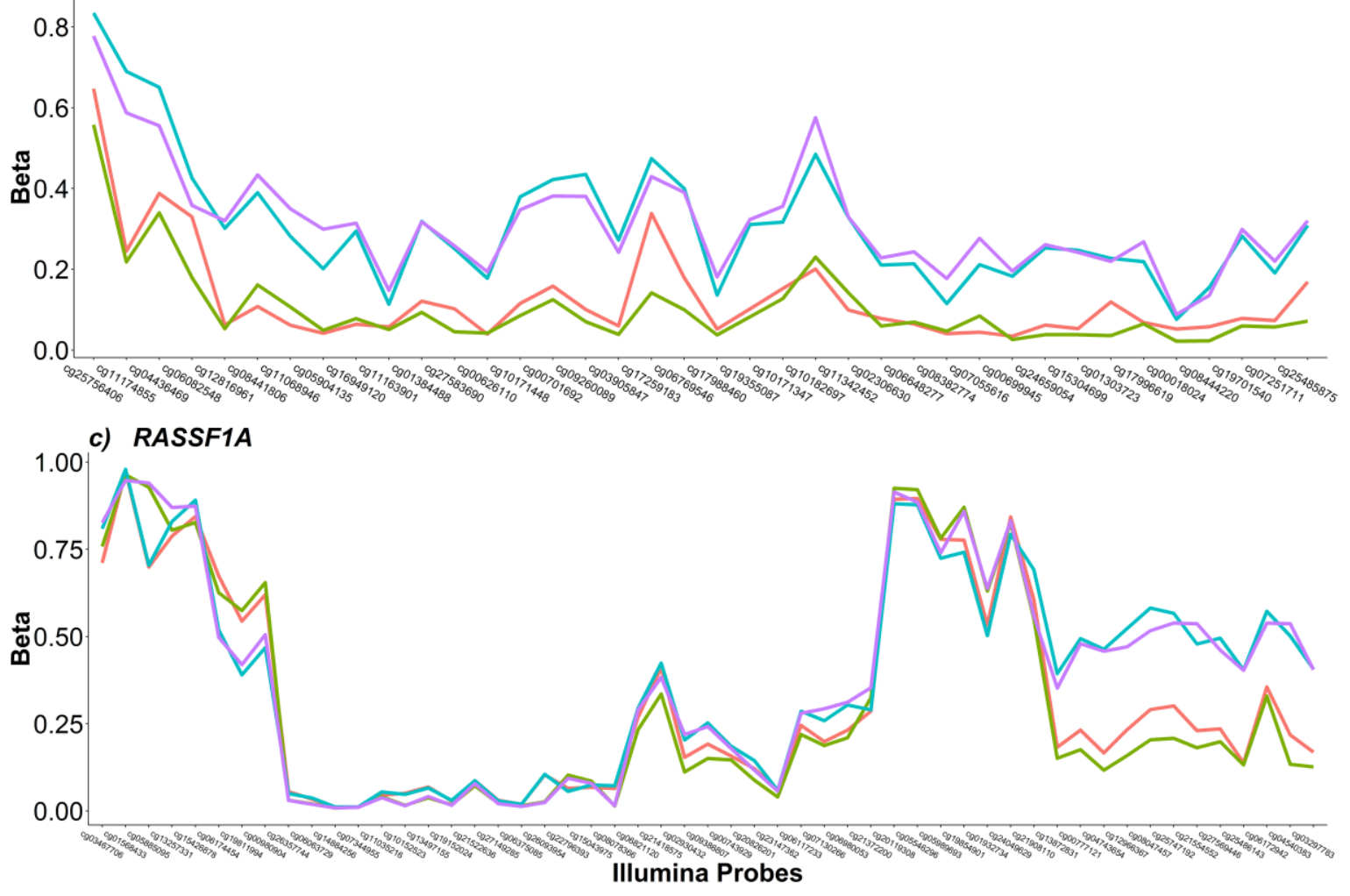

Sup Fig 2 

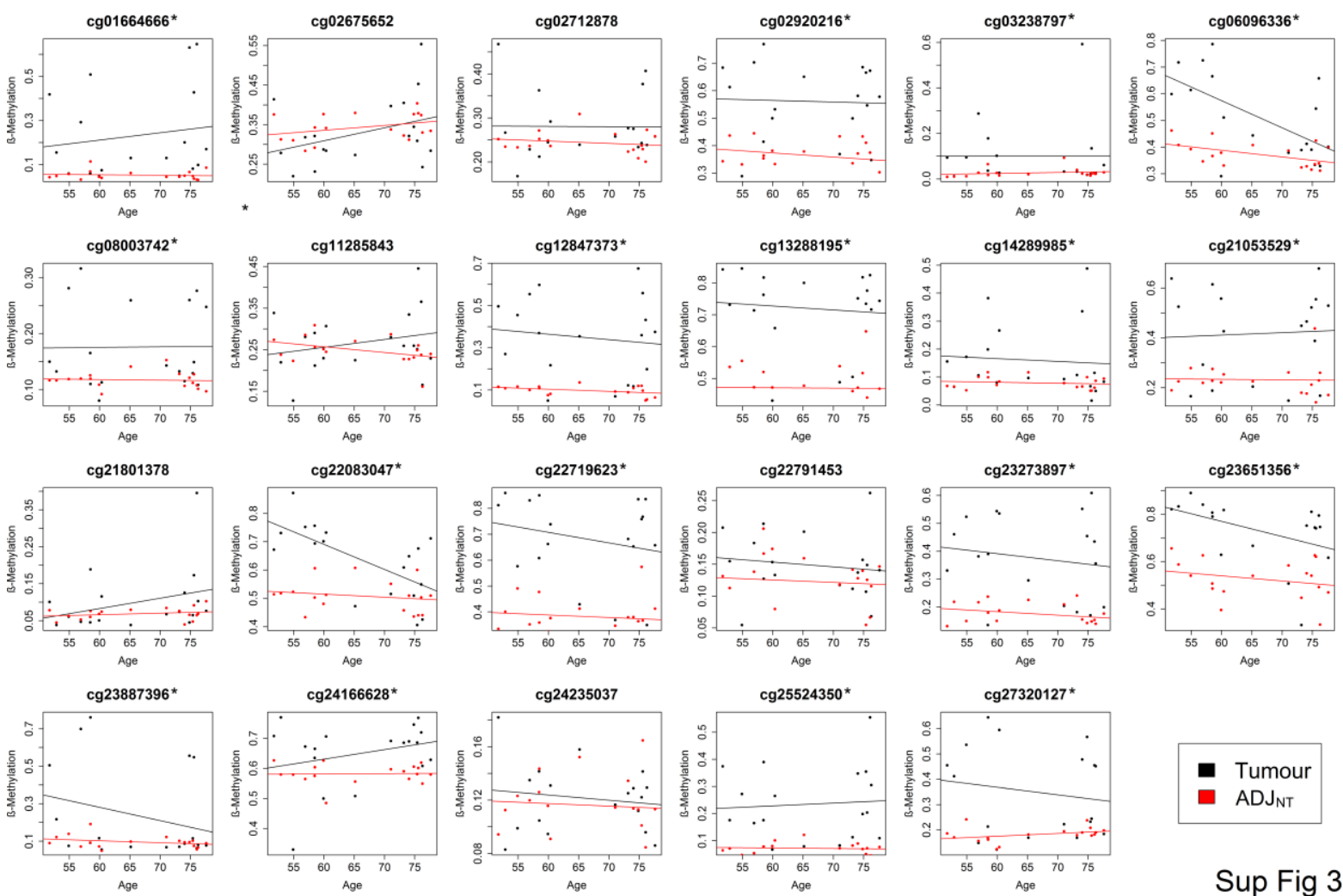

Tumour

ADJ $_{N T}$

Sup Fig 3 